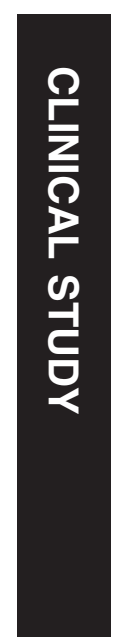

\title{
Sequential selective same-day suture removal in the management of post-keratoplasty astigmatism
}

${ }^{1}$ Division of Ophthalmology and Visual Sciences, University of Nottingham, Nottingham, UK

${ }^{2}$ Division of Ophthalmology and Visual Sciences, Nottingham University Hospitals, Queen's Medical Centre, Nottingham, UK

${ }^{3}$ Cornea and Ocular Surface Unit, Research Institute of Ophthalmology, Cairo, Egypt

Correspondence: HS Dua, Division of Ophthalmology and Visual Sciences, B floor, Research Room, Eye Ear Nose Throat Centre, Nottingham University Hospital, Queens Medical Centre,

Nottingham NG7 2UH, UK Tel: +44 (0)115924 9924; Fax: +44 (0)1159709963. E-mail: harminder.dua@ nottingham.ac.uk

Received: 29 March 2012 Accepted in revised form: 13 May 2013 Published online: 7 June 2013

\begin{abstract}
Aims In a previous study, we proposed that corneal topography performed $30-40 \mathrm{~min}$ after the initial suture removal can identify the next set of sutures requiring removal, for the treatment of post-keratoplasty astigmatism. The aim of this study was to evaluate the effect of removing subsequent sets of sutures at the same sitting. Methods 10/0 nylon interrupted sutures were placed, to secure the graft-host junction, at the time of keratoplasty. Topography was performed using Pentacam (Oculus) before suture removal. The sutures to be removed in the steep semi-meridians were identified and removed at the slit-lamp biomicroscope. Topography was repeated $30-40$ min post suture removal, the new steep semi-meridians determined, and the next set of sutures to be removed were identified and removed accordingly. Topography was repeated 4-6 weeks later and the magnitude of topographic astigmatism was recorded. A paired-samples $t$-test was used to evaluate the impact of selective suture removal on reducing the magnitude of topographic and refractive astigmatism.

Results Twenty eyes of 20 patients underwent sequential selective same-day suture removal (SSSS) after corneal transplantation. This study showed that the topographic astigmatism decreased by about $46.7 \%(3.68 \mathrm{D})$ and the refractive astigmatism decreased by about $37.7 \%$ (2.61 D) following SSSS. Vector calculations also show a significant reduction of both topographic and refractive astigmatism $(P<0.001)$

Conclusion SSSS may help patients to achieve satisfactory vision more quickly and
\end{abstract}

U Fares ${ }^{1,2}$, AA Mokashi², MS Elalfy ${ }^{1,2,3}$ and HS Dua ${ }^{1,2}$ reduce the number of follow-up visits required post keratoplasty.

Eye (2013) 27, 1032-1037; doi:10.1038/eye.2013.126; published online 7 June 2013

Keywords: astigmatism; penetrating keratoplasty; corneal topography; suture removal

\section{Introduction}

Post-keratoplasty astigmatism can adversely affect the visual outcome in an eye with a clear corneal graft. A number of different techniques have been described to address moderateto-high astigmatism following corneal transplantation. ${ }^{1}$ Suture manipulation is usually considered to be the first step in managing postkeratoplasty astigmatism, while the sutures are still present. This may include selective suture removal in the case of eyes with interrupted/ mixed sutures ${ }^{2}$ or suture adjustment in the case of eyes with running sutures. 3,4

The routine practice adopted is the removal of a pair of sutures at either end of the steep meridian (or one suture in the steep semimeridian), with reassessment of the refraction and topography after a period of 4-6 weeks, to ascertain which other sutures require removal. The process is repeated one or more times if further sutures require removal. This prolongs the visual rehabilitation of patients post keratoplasty. The use of hand-held keratoscopes for post-keratoplasty suture adjustment have been described, wherein it is recommended that a continuous suture can be adjusted at the slit-lamp until the keratoscope ring (reflection) becomes circular. It is also recommended that this can be used to identify which interrupted 
sutures can be removed. However, the modern approach is to identify the steep semi-meridians by topography. The authors have acknowledged this as a limitation of their simple approach. ${ }^{5}$ The effect of selective suture removal has been reported by several authors. ${ }^{2,6-8}$ Some controversy still surrounds the issue of removing one or more sets of sutures at a particular sitting. This relates to the lack of information on how soon after removal of interrupted suture(s) is the full effect observed, in terms of magnitude and vector of astigmatism. Removing additional sutures before the effect of the removal of the first set of sutures has settled could lead to wrong identification of the new steep semi-meridian(s) and the corresponding sutures to be removed. Furthermore, the effect of removal of more than one pair of sutures at one sitting on post-keratoplasty astigmatism is not well known. ${ }^{9,10}$

In general, tight sutures along the steep corneal semimeridians should be identified and removed. This can be achieved by clinical examination at the slit-lamp, refraction, keratometry or topography. Corneal topography has the advantage over refraction and keratometry in mapping subtle corneal power changes accurately over the entire optical zone. This allows identification of steep semi-meridians that can be attributed to specific sutures, which may not necessarily correspond to the refractive axis of astigmatism.

In a previous study, we proposed that corneal topography performed 30-40 min after the initial suture removal can identify the next set of sutures requiring removal. A second set of sutures can thus be removed at the same follow-up visit thereby reducing the number of follow-up visits and shortening the visual rehabilitation period (time to final spectacle or contact lens prescription). ${ }^{2}$

The aim of this study was to evaluate the effect of removing subsequent sets of sutures on post-keratoplasty astigmatism at the same sitting.

\section{Materials and methods}

This study included 20 consecutive post-keratoplasty eyes (20 patients) subject to selective suture removal to address astigmatism greater than 3 dioptres (D). This study was approved by the Queen's Medical Centre, University Hospitals NHS Trust, Nottingham, UK. Project number: 3117.

Inclusion criteria were post-keratoplasty astigmatism requiring suture removal; readable corneal topographic map; and post-operative period $>6$ months. Exclusion criteria were poor-quality topographic maps with insufficient data or the absence of sutures in the steep meridian.
Each patient followed a protocol of examination: medical history including indication for keratoplasty, suture technique, and operation; whether PK or triple procedure (PK combined with cataract extraction and intraocular lens implantation) and slit-lamp examination including intraocular pressure measurement.

Uncorrected visual acuity (UCVA), retinoscopy refraction and best-corrected visual acuity (BCVA) were recorded for all. In all eyes, 16 10/0 nylon interrupted sutures were placed, to secure the graft-host junction, at the time of keratoplasty. Topography was performed using Pentacam (Oculus, Optikgeräte $\mathrm{GmbH}$, Wetzlar, Germany) before suture removal. The sutures to be removed in the steep semi-meridians were identified and removed at the slit-lamp biomicroscope. Topography was repeated $30-40 \mathrm{~min}$ post suture removal, the new steep semi-meridians determined, and the next set of sutures to be removed were identified and removed accordingly. Topography was repeated 4-6 weeks later and the magnitude of topographic astigmatism was recorded. If further sutures required removal, the steep semi-meridians were identified and the same steps including further suture removal (as described above) were followed. At this point, only the topographic astigmatism was used for this study. The technique of suture removal has been described before. ${ }^{2}$ Patients were instructed to instil chloramphenicol $0.5 \%$ eye drops three times a day and predsol $0.5 \%$ eye drops three times a day for 1 week.

After suture removal, the changes in astigmatism were evaluated by the net reduction in astigmatism measured by topography and the calculation of the vector-corrected changes. Changes in visual acuity and complications after suture removal were also noted.

The following formula was applied for vector power calculations. This is well reported in the literature as an established method of astigmatic analysis: ${ }^{11,12}$

$$
\begin{aligned}
& M=S+(C / 2) \\
& \mathrm{J} 0=(-C / 2) \text { cosine }(2 \alpha) \\
& \mathrm{J} 45=(-C / 2) \text { sine }(2 \alpha)
\end{aligned}
$$

Where $M$, spherical equivalent refraction; $S$, sphere; $C$, cylinder; and $\alpha$, axis. The two-dimensional vector (J0, J45) was used to graphically analyse the astigmatic components of the vector powers.

\section{Statistical analysis}

All data were checked for normality using KolmogorovSmirnov test. A paired-samples $t$-test was used to evaluate the impact of selective suture removal on reducing the magnitude of topographic and refractive astigmatism. Statistical analysis was performed with the Statistical Package for the Social Sciences 19.0 for Windows (SPSS Inc., Chicago, IL, USA). 


\section{Results}

Twenty eyes of 20 patients underwent selective suture removal after corneal transplantation. The mean age of patients was 37 years (range 20-60 years). The mean follow-up from the date of keratoplasty to the time of first selective suture removal was 36 weeks (range 32-50 weeks). Clinical indications for keratoplasty are shown in Table 1.

\section{Topographic astigmatism}

The net reduction in the topographic astigmatism There was a statistically significant reduction in the magnitude of the topographic astigmatism regardless of the axis from $7.88 \pm 1.77 \mathrm{D}$ pre-suture removal to $5.90 \pm 1.52 \mathrm{D}$ after removing the first set of sutures (paired $t$-test, $P<0.001$ ). The mean decrease in the astigmatism was $1.98 \pm 0.63 \mathrm{D}$ (25\%) with a $95 \%$ confidence interval ranging from 1.68 to $2.27 \mathrm{D}$.

Notably, there was another significant decrease in the magnitude of the topographic astigmatism to $4.20 \pm 1.33 \mathrm{D}$ after removing the second set of sutures 30-40 min later (paired $t$-test, $P<0.001$ ). The mean decrease in the astigmatism was $1.70 \pm 0.34 \mathrm{D}(28.8 \%)$ with a $95 \%$ confidence interval ranging from 1.54 to $1.86 \mathrm{D}$.

Overall, the mean reduction of net astigmatism after removing both sets of sutures at the same follow-up visit was $3.68 \pm 0.70 \mathrm{D}(46.7 \%)$ with a $95 \%$ confidence interval ranging from 3.35 to $4.01 \mathrm{D}$ (paired $t$-test, $P<0.001$ ).

Table 1 Indications for keratoplasty

\begin{tabular}{lc}
\hline Surgery indication & Number \\
\hline Keratoconus & 8 \\
Pseudophakic bullous keratopathy & 5 \\
Microbial keratitis-related scars & 4 \\
Fuchs' endothelial dystrophy & 2 \\
Failed graft & 1 \\
\hline
\end{tabular}

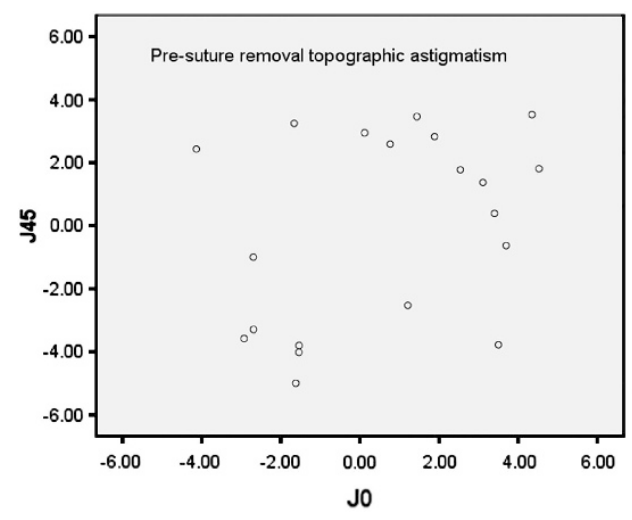

Vector-corrected change in topographic astigmatism after suture removal There was a statistically significant reduction in the magnitude of the topographic astigmatism with vector calculations from $3.94 \pm 0.89 \mathrm{D}$ pre-suture removal to $2.95 \pm 0.76 \mathrm{D}$ after removing the first set of sutures (paired $t$-test, $P<0.001$ ). The mean decrease in the astigmatism was $0.99 \pm 0.31 \mathrm{D}(25 \%)$ with a $95 \%$ confidence interval ranging from 0.84 to $1.12 \mathrm{D}$. Another significant decrease in the magnitude of the topographic astigmatism to $2.10 \pm 0.66 \mathrm{D}$ after removing the second set of sutures 30-40 min later (paired $t$-test, $P<0.001)$ was also noted. The mean decrease in the astigmatism was $0.85 \pm 0.17 \mathrm{D}(28.8 \%)$ with a $95 \%$ confidence interval ranging from 0.77 to $0.93 \mathrm{D}$.

Vector calculations showed a statistically significant reduction in the degree of astigmatism from 3.94 $\pm 0.89 \mathrm{D}$ (before removing the first set of sutures) to $2.10 \pm 0.66 \mathrm{D}$ (4-6 weeks after removing both sets of sutures) (paired t-test, $P<0.001)$. The mean decrease in the astigmatism was $1.84 \pm 0.35 \mathrm{D}(46.7 \%)$ with a $95 \%$ confidence interval ranging from 1.68 to $2.00 \mathrm{D}$ (Figure 1 ).

\section{Refractive astigmatism}

The net reduction in the refractive astigmatism Also, there was a statistically significant reduction in the magnitude of the refractive astigmatism regardless of the axis from $6.92 \pm 1.37 \mathrm{D}$ pre-suture removal to $4.31 \pm 1.37 \mathrm{D}$ after removing two sets of sutures at the same follow-up visit (paired $t$-test, $P<0.001)$. The mean reduction of the net refractive astigmatism was $2.61 \pm 0.93 \mathrm{D}(37.7 \%)$ with a $95 \%$ confidence interval ranging from 2.18 to $3.05 \mathrm{D}$.

Vector-corrected change in refractive astigmatism after suture removal Vector calculations showed a statistically significant reduction in the magnitude of astigmatism from $3.46 \pm 0.68 \mathrm{D}$ (before removing the first set of sutures) to $2.16 \pm 0.69 \mathrm{D}$ (4-6 weeks after removing two

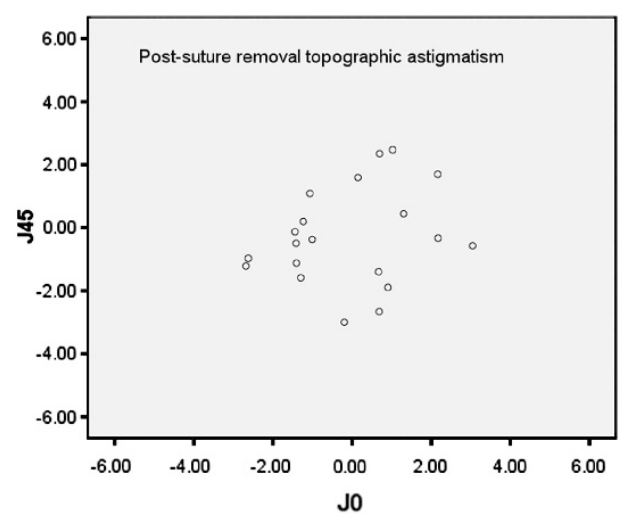

Figure 1 Topographic astigmatism before and after suture removal (dioptres), plotted as an astigmatic vector for each eye. 

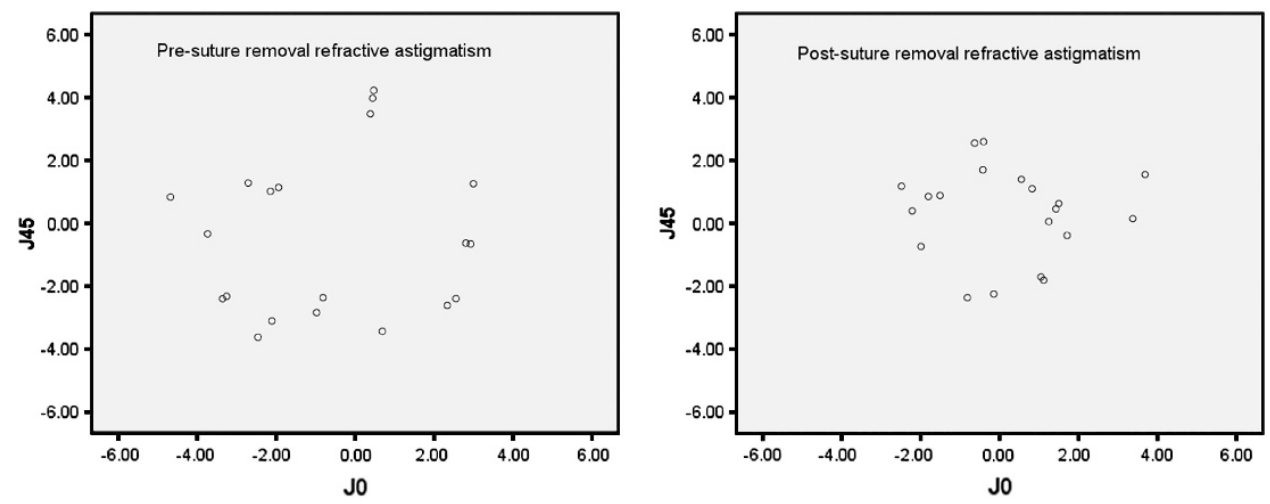

Figure 2 Refractive astigmatism before and after suture removal (dioptres), plotted as an astigmatic vector for each eye.

sets of sutures) (paired $t$-test, $P<0.001$ ). The mean decrease in the astigmatism was $1.31 \pm 0.47 \mathrm{D}(37.6 \%)$ with a $95 \%$ confidence interval ranging from 1.09 to $1.52 \mathrm{D}$ (Figure 2).

Figures 1 and 2 show clearly the collapse of the dispersed clusters towards the centre of the graph. This indicates a significant reduction in vector astigmatism change.

Visual acuity changes associated with suture removal BCVA improved in 12 cases (60\%), did not change in 7 cases (35\%), and decreased in 1 case (5\%) 4-6 weeks after removing subsequent sutures. On the other hand, UCVA improved in 9 cases, did not change in 9 cases $(45 \%)$, and decreased in 2 cases (10\%).

None of the patients included in our study had any complications including infections, wound dehiscence, or rejection following sequential selective same-day suture removal (SSSS).

\section{Discussion}

A clear corneal graft is not the only criterion for successful keratoplasty. Management of postkeratoplasty astigmatism is a major challenge for corneal and refractive surgeons. A number of factors influence astigmatism after corneal transplant surgeries. This includes donor and recipient factors such as trephination, graft-host size disparity, wound healing and apposition, suturing technique, and suture material. ${ }^{1}$

Different techniques are described to secure the grafthost junction. This includes double or single running sutures, interrupted sutures, and combined running and interrupted sutures. The use of the latter two techniques allows for selective suture removal of the interrupted sutures. The concept of selective suture removal was first introduced by Cottingham. ${ }^{13}$ Later, many authors adopted this technique to address post-keratoplasty astigmatism and reported variable results. ${ }^{9,14,15}$
Nylon sutures are the most commonly used suture material to secure donor buttons in penetrating and deep anterior lamellar keratoplasty. At a variable time point post surgery, suture management of astigmatism is commenced. This is early ( $<1$ to 3 months) with doublecontinuous or combined continuous and interrupted sutures and later (3-4 months) with interrupted sutures only. Once astigmatism is brought down to a manageable level with good vision, a variable number of sutures are left in situ. 8,9 Whether all sutures should be removed at some point or not remains a dilemma for all corneal surgeons. No matter when all sutures are removed, refractive surprises can occur, adversely affecting the patients' vision. ${ }^{7}$ Equally, leaving sutures in place carries the risk of suture breakage with risk of new vessels, infection, and rejection. When the pros and cons are discussed with the patient, it is not surprising that some prefer to leave the remaining sutures retaining their 'good' vision and others opt to have them removed.

How many sutures can be safely removed in one sitting without risking graft dehiscence depends on a number of variables. By 6 months after surgery, most wounds are secure enough to allow removal of one or more pairs of interrupted sutures. An important point to consider is to avoid pulling the suture knot through the graft-host junction during suture removal. We have noted that the risk of dehiscence of the graft-host junction during or immediately after suture removal is higher in women over 70 years (unpublished observation). In the context of selective removal of sutures for management of astigmatism, it is generally accepted that a pair of opposing sutures in the steep semi-meridian be removed in any one sitting. This can be extended to three or four sutures in steep semimeridians. ${ }^{1,2,12}$ The rationale for this is that the effect of suture removal on the astigmatism is not instant both in terms of magnitude and vector, and a reassessment for residual astigmatism and axis was therefore undertaken between 3 and 6 weeks after removal of one pair (or four 
sutures). ${ }^{1,2,8}$ When suture removal is commenced in the early post-operative period there is an additional risk of wound dehiscence if adjacent sutures are removed, especially in the elderly who would also be on topical steroids, which are known to delay wound healing. ${ }^{1}$ Furthermore, it has been shown that removing a single suture was more efficient and predictable than removing multiple sutures at one time. ${ }^{10}$

In our previous study, topography maps showed that the axis of the steep semi-meridians did not shift more than 22.5 degrees, between 30-40 min and 4-6 weeks after removing only one set of sutures, in $85 \%$ of the eyes. Moreover, we showed that there was no significant difference between the mean net astigmatism 30-40 min after removing one set of sutures (4.37D) and the mean net astigmatism 4-6 weeks later, without removing any additional sets (4.24D). On this basis, we had suggested that removing a second set of sutures at the same follow-up visit would expedite post-keratoplasty visual rehabilitation and reduce the number of follow-up visits. ${ }^{2}$

In this study, we undertook what we had suggested and evaluated the outcome. In our previous study (20 eyes), there was also a significant decrease in the topographic astigmatism $(33.3 \%, 2.12$ D) $4-6$ weeks after removing only one set of sutures. ${ }^{2}$ On the other hand, this study showed that the topographic astigmatism decreased by about $46.7 \%$ (3.68 D) following SSSS. In both studies, topographic steep semi-meridians were used to identify tight sutures. However, the effect of suture removal has been shown to be greater when there is an agreement between refractive and topographic axes. ${ }^{16}$

In general, the aim of post-keratoplasty astigmatism management by selective suture removal is to enable patients to achieve a satisfactory BCVA, either with spectacles or with contact lenses. In our study, both topography and refractive astigmatism were significantly decreased after sequentially removing two sets of sutures. This was associated with an improvement in the BCVA and the UCVA.

SSSS helps to decrease the magnitude of astigmatism and render it more regular. This explains why the BCVA and the UCVA improved in 60 and $45 \%$ of the eyes, respectively. No eye lost more than one line of UCVA Snellen visual acuity and only one eye (5\%) lost one line of Snellen BCVA. Failure of improvement in the other cases could be related to residual irregular astigmatism.

Although Strelow et al ${ }^{10}$ reported that removing a single suture was more efficient and predictable than removing multiple sutures at one time, they were unable to separate the confounding variable of pre-existing irregular astigmatism. Also, modern and recent topography machines, such as the Pentacam, give more details about the corneal topographic map and thus enable corneal surgeons to be more precise in their selective suture removal.

Binder ${ }^{17}$ studied the change in astigmatism after removal of all sutures in 188 cases and found that leaving sutures in place for 18 months or more appears to provide a more stable wound and less corneal curvature change after late suture removal. If sutures are removed well before the wound has healed, unpredictable results are more likely to occur.

Burk et $a l^{18}$ reported a mean change of corneal astigmatism of 2.0-3.0 D after removing one to four interrupted sutures. They reported a variable change of astigmatism, $54 \%$ of their cases had a reduction of astigmatism by $\geq 1.00 \mathrm{D}$ and $18 \%$ had an increase of astigmatism by $\geq 1.00 \mathrm{D}$. This was explained by the lack of good techniques to identify tight sutures. In our series, the net topographic and refractive astigmatism reduction was 3.68 and $2.61 \mathrm{D}$, respectively.

How many sutures can be removed at the same visit is an interesting and important question. The longer the interval between surgery and suture removal, the less likely are the chances of wound-related problems, particularly in elderly patients on long-term topical steroids. Removal of adjacent sutures is more likely to stress the wound than removal of alternate or nonadjacent sutures. If a combined running and interrupted suturing technique is used, many of the interrupted sutures can be safely removed with minimal risk of wound-related problems. Nylon sutures degrade with time and late removal (over a year) can be associated with suture breakage and incomplete removal. Every episode of suture removal carries the added risk of infection and/or graft rejection. Appropriate antibiotic and steroid cover is therefore essential.

If two sets of sutures can be safely removed at a given follow-up visit, how many sets could be removed at the subsequent follow-up visit? Many factors have a role in the answer to this question, such as the age of patient, time elapsed after surgery, and the location of the second set of sutures. If the magnitude of astigmatism is still high, at least one further set of sutures can be removed. Further suture removal at the same visit should be made on a case by case basis.

This study constitutes a further step forward in understanding of the effect of selective suture removal on post-keratoplasty astigmatism. This technique may help patients to achieve satisfactory vision more quickly and reduce the number of follow-up visits. This latter factor has economic implications in both public and private sectors. Further studies investigating the role of SSSS in the management of post-keratoplasty (both PK and DALK) astigmatism would be of value. 
The long-term risks of leaving sutures in situ have been discussed above. Some surgeons advocate removal of all sutures at 1-2 years post-operatively. ${ }^{19}$ The only reason for not removing all sutures at a given point in time is the refractive (usually astigmatic) surprise and consequent poor vision with the additional risk, however small, of inducing a rejection or infection episode consequent upon removal of multiple sutures. Some surgeons therefore leave the suture for much longer periods of time or indefinitely. Mader et $a l^{7}$ have reported a major change in dioptre and axis of astigmatism following suture removal 1-6 years post keratoplasty. Our practice is to present the patient with the pros and cons and offer a choice. Many opt for good vision with retained sutures but are warned to attend the eye casualty department at any symptom in the grafted eye. The long-term follow-up of patients with SSSS, comparing those with and without removal of all sutures, will yield useful information.

\section{Summary}

\section{What was known before}

- Many studies reported the effect of selective suture removal to address post-keratoplasty astigmatism. We showed in a previous paper published in Eye 2010 that topography can predict the next set of sutures requiring removal. The effect of removing more than one set of sutures is not yet well known.

\section{What this study adds}

- We report the effect of sequential same-day suture removal. This study constitutes a further step forward in understanding of the effect of selective suture removal on post-keratoplasty astigmatism. This technique may help patients to achieve satisfactory vision more quickly and shorten the number of follow-up visits.

\section{Conflict of interest}

The authors declare no conflict of interest.

\section{References}

1 Fares U, Sarhan AR, Dua HS. Management of post-keratoplasty astigmatism. J Cataract Refract Surg 2012; 38(11): 2029-2039.

2 Sarhan AR, Fares U, Al-Aqaba MA, Miri A, Otri AM, Said DG et al. Rapid suture management of post-keratoplasty astigmatism. Eye 2010; 24(4): 540-546.
3 Hovding G. Suture adjustment in penetrating keratoplasty. Acta Ophthalmol 1994; 72(2): 246-252.

4 Roper-Hall MJ. Adjustment of running suture to control postkeratoplasty astigmatism. Ophthalmology 1991; 98(5): 561.

5 Allan BD, Barrett GD. Qualitative keratoscopy at the slitlamp in postkeratoplasty suture adjustment. I Cataract Refract Surg 1993; 19(6): 817.

6 Krumeich J, Binder PS, Knulle A. The theoretical effect of trephine tilt on postkeratoplasty astigmatism. CLAO J 1988; 14(4): 213-219.

7 Mader TH, Yuan R, Lynn MJ, Stulting RD, Wilson LA, Waring 3rd, GO. Changes in keratometric astigmatism after suture removal more than one year after penetrating keratoplasty. Ophthalmology 1993; 100(1): 119-126; discussion 27.

8 Van Meter WS, Gussler JR, Soloman KD, Wood TO. Postkeratoplasty astigmatism control. Single continuous suture adjustment versus selective interrupted suture removal. Ophthalmology 1991; 98(2): 177-183.

9 Binder PS. Selective suture removal can reduce postkeratoplasty astigmatism. Ophthalmology 1985; 92(10): 1412-1416.

10 Strelow S, Cohen E, Leavitt K, Laibson P. Corneal topography for selective suture removal after penetrating keratoplasty. Am J Ophthalmol 1991; 112: 657-665.

11 Nubile M, Carpineto P, Lanzini M, Calienno R, Agnifili L, Ciancaglini $\mathrm{M}$ et al. Femtosecond laser arcuate keratotomy for the correction of high astigmatism after keratoplasty. Ophthalmology 2009; 116(6): 1083-1092.

12 Poole TR, Ficker LA. Astigmatic keratotomy for postkeratoplasty astigmatism. J Cataract Refract Surg 2006; 32(7): 1175-1179.

13 Cottingham AJ. Residual astigmatism following keratoplasty. Ophthalmology 1980; 87(suppl): 113-114.

14 Stainer GA, Perl T, Binder PS. Controlled reduction of postkeratoplasty astigmatism. Ophthalmology 1982; 89(6): 668-676.

15 Feldman ST, Brown SI. Reduction of astigmatism after keratoplasty. Am J Ophthalmol 1987; 103: 477-478.

16 Sarhan AR, Dua HS, Beach M. Effect of disagreement between refractive, keratometric, and topographic determination of astigmatic axis on suture removal after penetrating keratoplasty. $\mathrm{Br} J$ Ophthalmol 2000; 84: 837-841.

17 Binder PS. The effect of suture removal on postkeratoplasty astigmatism. Am J Ophthalmol 1988; 106: 507.

18 Burk LL, GOr Waring, Radjee B, Stulting RD. The effect of selective suture removal on astigmatism following penetrating keratoplasty. Ophthalmic Surg 1988; 19: 849-854.

19 Serdarevic ON, Renard GJ, Pouliquen Y. Randomized clinical trial of penetrating keratoplasty. Before and after suture removal comparison of intraoperative and postoperative suture adjustment. Ophthalmology 1995; 102(10): 1497-1503. 